\title{
Uniquely Hamiltonian Characterizations of Distance-Hereditary and Parity Graphs
}

\author{
Terry A. McKee \\ Department of Mathematics \& Statistics \\ Wright State University, Dayton, Ohio 45435 USA \\ terry.mckee@wright.edu
}

Submitted: Apr 9, 2007; Accepted: Sep 19, 2008; Published: Sep 29, 2008

Mathematics Subject Classifications: 05C75, 05C45

\begin{abstract}
A graph is shown to be distance-hereditary if and only if no induced subgraph of order five or more has a unique hamiltonian cycle; this is also equivalent to every induced subgraph of order five or more having an even number of hamiltonian cycles. Restricting the induced subgraphs to those of odd order five or more gives two similar characterizations of parity graphs. The close relationship between distancehereditary and parity graphs is unsurprising, but their connection with hamiltonian cycles of induced subgraphs is unexpected.
\end{abstract}

\section{Distance-hereditary graphs}

Howorka [10] defined a graph to be a distance-hereditary graph if the distance between vertices in connected induced subgraphs always equals the distance between them in the full graph. This is equivalent to every cycle of length five or more having two crossed chords; $[1,4,7,9]$ contain many additional characterizations.

Lemma 1 will state two characterizations of distance-hereditary graphs from [1] that will be used below. As in [4], the vertices in a set $S \subset V(G)$ are twins if they all have exactly the same neighbors in $G-S$ and are either pairwise adjacent (in which case they are true twins) or pairwise nonadjacent (in which case they are false twins). A pendant vertex is a vertex $v$ for which there is a vertex $w$ such that $N(v)=\{w\}$; the edge $v w$ is then a pendant edge. A graph $G^{\prime}$ is a one-vertex expansion [1] of a graph $G$ if $V\left(G^{\prime}\right)=V(G) \cup\left\{v^{\prime}\right\} \neq V(G)$ where either $v^{\prime}$ has a twin vertex $v$ in $G$ or $v^{\prime}$ is a pendant vertex of $G^{\prime}$.

Lemma 1 (Bandelt \& Muller 1986) Each of the following is equivalent to $G$ being a distance-hereditary graph: 
(1.1) Each component of $G$ is built from a single vertex by a sequence of one-vertex expansions.

(1.2) $G$ contains no induced cycle of length five or more and no induced house, gem, or domino subgraph (see Figure 1).
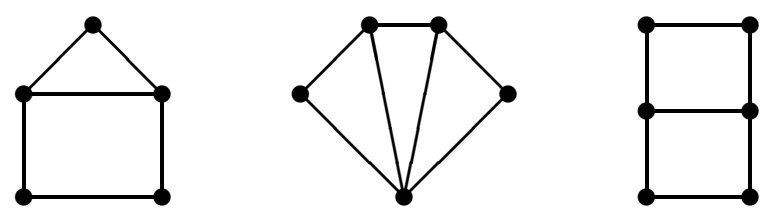

Figure 1: From left to right, the house, gem, and domino graphs.

Theorem 2 presents two new characterizations of distance-hereditary graphs. A graph is uniquely hamiltonian [3] if it has a unique hamiltonian cycle (see [8] for updated discussion).

Theorem 2 Each of the following is equivalent to being distance-hereditary:

(2.1) No induced subgraph of order five or more is uniquely hamiltonian.

(2.2) Every induced subgraph of order five or more has an even number of hamiltonian cycles.

Proof. First suppose $G$ is a distance-hereditary graph [toward showing (2.2)]. Using (1.1) of Lemma 1 and arguing by induction, suppose a connected graph $G$ satisfies (2.2) and $G^{\prime}$ is a one-vertex expansion of $G$ with $V\left(G^{\prime}\right)=V(G) \cup\left\{v^{\prime}\right\}$ [toward showing that $G^{\prime}$ also satisfies (2.2)]. Suppose $H$ is any induced subgraph of $G^{\prime}$ of order five or more, noting that if $H$ has no spanning cycle then $H$ has zero hamiltonian cycles. So suppose $H$ has a spanning cycle $C$ [toward showing that $H$ has an even number of hamiltonian cycles]. If $v^{\prime}$ is a pendant vertex of $G^{\prime}$, then $v^{\prime} \notin V(C)=V(H)$, so $H$ is an induced subgraph of $G$ and so has an even number of hamiltonian cycles. Suppose $v^{\prime}$ is the twin of some $v \in V(G)$. If $v^{\prime} \notin V(H)$, then $H$ has an even number of hamiltonian cycles since $H$ is an induced subgraph of $G$. If $v^{\prime} \in V(H)$ but $v \notin V(H)$, then $H$ has an even number of hamiltonian cycles since $G^{\prime}-v^{\prime} \cong G$. So suppose $H$ contains both the twins $v$ and $v^{\prime}$. Say that $C$ is 'equivalent' to any spanning cycle $C^{\prime}$ of $H$ for which $v x \in E(C) \Leftrightarrow v^{\prime} x \in E\left(C^{\prime}\right)$ for every $x \in V(H)$. Doing this over all the spanning cycles of $H$ produces equivalence classes of cardinality two. Therefore, there must be an even number of such spanning cycles $C$, which means that $H$ has an even number of hamiltonian cycles.

Therefore, being a distance-hereditary graph implies condition (2.2). Condition (2.2) trivially implies (2.1). Condition (2.1) implies being a distance-hereditary graph by contraposition, since each of the forbidden subgraphs described in (1.2) is uniquely hamiltonian of order five or more.

Theorem 2 essentially says: A graph is a distance-hereditary graph if and only if every uniquely hamiltonian induced subgraph is isomorphic to $C_{3}, C_{4}$, or $K_{1,1,2}\left(\cong K_{4}\right.$ with an edge deleted). 
A block of a graph is a maximal nonseparable subgraph. A graph in which every block is complete is frequently called a block graph (or sometimes a Husimi tree or a completed Husimi tree); see [4] for characterizations. Every block graph is distance-hereditary, but $C_{4}$ is a distance-hereditary graph that is not a block graph.

Corollary 3 Every block of a graph is complete if and only if:

(3.1) No induced subgraph of order four or more is uniquely hamiltonian.

Every block of a graph is isomorphic to $K_{2}$ or $K_{3}$ if and only if:

(3.2) Every induced subgraph of order four or more has an even number of hamiltonian cycles.

Proof. First suppose every block of $G$ is complete. Then $G$ is a distance-hereditary graph and so, by (2.1), no induced subgraph of $G$ of order five or more is uniquely hamiltonian. The only order- 4 graphs that are uniquely hamiltonian $\left(C_{4}\right.$ and $\left.K_{1,1,2}\right)$ fail to have every block complete. Therefore, no induced subgraph of $G$ of order four or more is uniquely hamiltonian.

Conversely, if $G$ has a block that is not complete, then $G$ either contains an induced cycle $C_{k}$ with $k \geq 4$ or an induced $K_{1,1,2}$. In either case, $G$ contains an induced subgraph of order four or more that is uniquely hamiltonian.

The second part of the corollary is proved similarly, noting the following:

(1) The only order-4 graphs with an odd number of hamiltonian cycles $\left(C_{4}, K_{1,1,2}\right.$, and $K_{4}$ ) fail to have every block isomorphic to $K_{2}$ or $K_{3}$.

(2) A graph with a block that is not isomorphic to $K_{2}$ or $K_{3}$ has an induced cycle $C_{k}$ with $k \geq 4$ or an induced $K_{1,1,2}$ or $K_{4}$, and so has an induced subgraph of order four or more that has an odd number of hamiltonian cycles.

By comparison, graph is a forest if and only if no induced subgraph of order three or more is uniquely hamiltonian, which is also equivalent to every induced subgraph of order three or more having an even number (zero) of hamiltonian cycles. In other words, a graph is a forest if and only if no induced subgraph is uniquely hamiltonian.

\section{Parity graphs}

Burlet \& Uhry [5] defines a graph to be a parity graph if, for every pair of vertices, the lengths of all the induced paths between them have the same parity. This is equivalent to every cycle of odd length five or more having two crossed chords; $[2,4,6]$ contain additional characterizations. Every distance-hereditary graph is a parity graph, but the domino graph (see Figure 1) is a parity graph that is not a distance-hereditary graph.

Lemma 4 will state two characterizations of parity graphs from [5] that will be used below. Suppose $G$ is any graph with false (independent) twins $x_{1}, \ldots, x_{n}(n \geq 1)$ and $B$ is any bipartite graph vertex disjoint from $G$ that contains vertices $y_{1}, \ldots, y_{n}$ from a common color class. As in [5], a vertex extension $G^{\prime}$ of $G$ by a bipartite graph $B$, with respect to the extension vertices $x_{1}, \ldots, x_{n}$ results from identifying the vertices $x_{i}$ and $y_{i}$ 
for each $i \leq n$. (The vertex extension by $B \cong K_{2}$ with respect to $n=1$ extension vertex is the one-vertex expansion that has $B$ a pendant edge.) A short chord of a cycle $C$ is an edge $u w$ where $u$ and $w$ are a distance two apart along $C$.

Lemma 4 (Burlet \& Uhry 1984) Each of the following is equivalent to $G$ being a parity graph:

(4.1) Each component of $G$ is built from a single vertex by a sequence of one vertex expansions and vertex extensions by bipartite graphs.

(4.2) G contains no induced cycle of odd length five or more, possibly with a unique short chord, and no induced gem subgraph (see Figure 1).

Theorem 5 Each of the following is equivalent to being a parity graph:

(5.1) No induced subgraph of odd order five or more is uniquely hamiltonian.

(5.2) Every induced subgraph of odd order five or more has an even number of hamiltonian cycles.

Proof. First suppose $G$ is a parity graph [toward showing (5.2)]. Using (4.1) of Lemma 4 and arguing by induction, suppose a connected graph $G$ satisfies (5.2) and $G^{\prime}$ is either a one vertex expansion of $G$ or a vertex extension of $G$ by a bipartite graph [toward showing that $G^{\prime}$ also satisfies (5.2)]. Suppose $H$ is any induced subgraph of $G^{\prime}$ of odd order five or more, noting that if $H$ has no spanning cycle then $H$ has zero hamiltonian cycles. So suppose $H$ has a spanning cycle $C$ [toward showing that $H$ has an even number of hamiltonian cycles]. If $V(C) \subseteq V(G)$, then $H$ is an induced subgraph of $G$ and so has an even number of hamiltonian cycles. So suppose $V(C) \nsubseteq \subseteq V(G)$. If $G^{\prime}$ is a one vertex expansion of $G$, then argue precisely as in the proof of Theorem 2 .

Suppose $G^{\prime}$ is a vertex extension by a bipartite graph $B$ with respect to extension vertices $x_{1}, \ldots, x_{n}$. Reorder the subscripts if necessary so that $x_{1}, \ldots, x_{k}$ are those vertices at which $C$ passes between $G$ and $B$. (It is possible to have $k<n$ because an extension vertex could have both its edges along $C$ in $G$ or both in $B$.) For each $1 \leq i \leq k$, let $w_{i}$ be the neighbor of $x_{i} \in V(G)-V(B)$ along $C$. Say that $C$ is 'equivalent' to any spanning cycle $C^{\prime}$ of $H$ that uses the same sets $\left\{x_{1}, \ldots, x_{k}\right\}$ and $\left\{w_{1}, \ldots, w_{k}\right\}$. Since $x_{1}, \ldots, x_{n}$ are false twins in $G$, if edges $x_{i} w_{i}$ and $x_{i+1} w_{i+1}$ are in a common path along $C$ within $G$, then those two edges can be replaced with $x_{i} w_{i+1}$ and $x_{i+1} w_{i}$ to form a distinct equivalent cycle $C^{\prime}$. Doing this over all the spanning cycles of $H$ produces equivalence classes of even cardinality. Therefore, there must be an even number of such spanning cycles $C$, which means that $H$ has an even number of hamiltonian cycles.

Therefore, being a parity graph implies condition (5.2). Condition (5.2) trivially implies (5.1). Condition (5.1) implies being a parity graph by contraposition, since each of the forbidden subgraphs described in (4.2) is uniquely hamiltonian of odd order five or more.

Theorem 5 essentially says: A graph is a parity graph if and only if its only uniquely hamiltonian induced subgraphs are triangles. 
By comparison, notice that a graph is bipartite if and only if no induced subgraph of odd order is uniquely hamiltonian, which is also equivalent to every induced subgraph of odd order having an even number (zero) of hamiltonian cycles. (This follows from Theorem 5, using that $C_{3}$ is the only order-3 hamiltonian graph and that every nonbipartite graph contains an induced cycle $C_{k}$ with $k$ odd.)

\section{References}

[1] H.-J. Bandelt and H. M. Mulder. Distance-hereditary graphs. J. Combin. Theory (Ser. B) 41 (1986) 182-208.

[2] H.-J. Bandelt and H. M. Mulder. Metric characterization of parity graphs. Discrete Math. 91 (1991) 221-230.

[3] C. A. Barefoot and R. C. Entringer, Extremal maximal uniquely Hamiltonian graphs, J. Graph Theory 4 (1980) 93-100.

[4] A. Brandstädt, V. B. Le, and J. P. Spinrad, Graph Classes: A Survey, Society for Industrial and Applied Mathematics, Philadelphia, 1999.

[5] M. Burlet and J.-P. Uhry, Parity graphs, in Topics on Perfect Graphs [C. Berge and V. Chvátal, eds., North-Holland, Amsterdam] Ann. Discrete Math. 21 (1984) 253-277.

[6] S. Cicerone and G. Di Stefano, On the extension of bipartite to parity graphs, Discrete Appl. Math. 95 (1999) 181-195.

[7] A. D'Atri and M. Moscarini. Distance-hereditary graphs, Steiner trees, and connected domination. SIAM J. Comput. 17 (1988) 521-538.

[8] R. J. Gould, Advances on the Hamiltonian problem-a survey, Graphs Combin. 19 (2003) 7-52.

[9] P. L. Hammer and F. Maffray. Completely separable graphs. Discrete Appl. Math. 27 (1990) 88-99.

[10] E. Howorka. A characterization of distance-hereditary graphs. Quart. J. Math. Oxford (Ser. 2) 28 (1977) 417-420. 